\title{
ON THE NUMBER OF RGB COLOURS WE CAN DISTINGUISH PARTITION SPECTRA
}

\author{
E. LÁBOS \\ Research Group of Neurobiology of Hungarian Academy of Sciences \\ at the Semmelweis University \\ 1094 Budapest, Tüzoltó $u .58$. \\ (phone: +36-1-215-6920/3632; fax: +36-1-215-5158) \\ E. Lábos \\ e-mail:labos@anal.sote.hu \\ (Received $10^{\text {th }}$ Sep 2005, accepted $10^{\text {th }}$ Oct 2006)
}

\begin{abstract}
Colour vision is a physiological phenomenon, and rays are not coloured (Newton, Wright). While counting hues of solar spectrum is a solved problem, the exact number of colours is unknown. Existing colour systems do not provide automatically colour census methods.

This work addressed only to the 24 bit RGB-colour system. This complete colour space itself is countable. Discovering principles for ordering colours is a prerequisite of any efficient census algorithm. Colours (r, g, b) we define, as a partition of total $n=r+g+b$ amount of intensity into 3 parts also with $r / g$ and $g / b$ ratios fixed. Partition spectra are the lexicographically sorted list of these partitions. Such and also geometrically defined sub-domains of RGB-cube can be transformed into 1D colour spectra by reshuffling according to their HSV-hue-angles in order to bring similar colours into close neighbourhood. These we call pseudo-solar colour spectra. Our estimation for subjectively discriminated RGB-colours is no more than 200000.
\end{abstract}

Keywords: partition-colour-spectra, RGB-cube, HSV-space, ordering colours, reshuffling by hues.

\section{Introduction}

To distinguish numerous colours is an ancient experience. The number of hues in rainbow is one of the first related knowledge, associated with Aristotle and Newton [1]. Colour sensation is physiologically founded. No physical arguments for segregation of zones inside the visible electromagnetic continuum exist. Rainbow only appear to be divided. Thus colour is a permanent illusion of normal human subjects. Rays are not coloured (Newton,Wright, [2]). Thus census of colours is finally a human experimental issue. However, preparation of enumerations, that is our actual purpose, should and can be supported with mathematical tools

In any artificial representations, colours are arranged by some regular way in a bounded domain of 1, 2 or 3 dimensions. Inside, points are usually described with 1, 2 or 3 parameters. Such colour-spaces are based on some colour theory. Geometrically, that spaces are either linear spectra, circles, wheels, triangles, cubes, cones, pyramids, cylinders, matrices (charts) or more sophisticated manifolds. Nearly hundred of such historical colour-systems have been constructed. Handbooks like [3] and [4] give good surveys. An incomplete, arbitrary selection may include names from Aristotle to Munsell. While the most important modern colour systems are CIE1931, RGB, HSV [5] and CMYK (see Glossary). 
Neither of colour-systems offers perfect solution for the precise counting of colours or hues we can discriminate. However, all ones provide significant help to approach the answer.

Present considerations are restricted to RGB and partly deals with to HSV system [5].

The RGB model is used to describe colours of computer monitor emissions in a digital fashion. An RGB-colour is a (red, green, blue) vector. Components are either between 0 and 1 or integers between 0 and 255. RGB-system is in close connection with the very consolidated tristimulus colour-vision theory of Young - Helmholtz - Maxwell for human. It is based on 3 cones with maximal sensitivity at 564, 534 and $420 \mathrm{~nm}$ supplemented with brightness channel of rods. Different facts hold to other animals. In monitors RGB system describes screen emission of 3 primary colour components through 24 bits per pixel information. Further colours are derived by additive colour mixing.

Intensive use of monitors keeps alive the misbelief that we might distinguish all the $2^{24}=16777216$ distinct colours simply because the screen display them.

Counting of hues is different from that of colours and it is easier. Here we accept, as the $1^{\text {st }}$ definition of hue that one-hue $=$ one-wavelength. Numerous less precise definitions of hue like dominant wavelength, colour-quality, name are not always useful or even misleading. Set of hues becomes finite and countable if perceivable ranges are determined. The estimate for the count of discriminable, almost-monochromatic spectral colours, the hues, arises from psychophysical studies of wavelength discriminationthreshold curves $[6,7,8,9]$. In 400-800 nm $\lambda$-range usually more than 1 , often 2,3 , sometimes 6-8 nm $\Delta \lambda$-shift is required (in average 3.1-3.3 nm) to perceive a different hue. Averaging available $\Delta \lambda / \lambda$ curves of Wright and Pitt [6]. approximately 100-120 narrow, i.e. quasi-monochromatic spectral light-ranges ( = practical hues) we can encounter or distinguish.

In HSV-systems [5], colours are specified through properties like hue, saturation, and lightness (or value). HSV system provides a $2^{\text {nd }}$ definition of hue as a computed quantity from parameters of other colour systems. In RGB - system, magnitudes of $r, g$ and $b$, the so called primary components hide values of brightness and saturation and hue. Several conversion formulas between RGB and HSV are available [5]. In HSV, circular huespectra is applied., essentially a solar spectrum., closed into a circle.

Further colour-counts are based upon colour charts, collections, catalogues, atlas for painters, printers or recently for web-sites or monitors. Nemcsics [3] and Lukács [4] provide large list of historically important tables of colour - collections. The number of colour in these various catalogues lays between 94 and 25000. Such a list appeared already 450 years ago.

In the CIE1931 diagram, discrimination or MacAdam ellipses or tolerance - spheres serve to separate equivalence classes of colours $[10,3,4]$.

It is not the main problem is how to harmonize 7 rainbow-colours, 120 spectral hues, 100 - 25000 catalogue items or 16777216 colours of RGB system. The true problem is the suitable order of colours. However, colour identification is rendered more difficult by additional factors.

- (1\#)Various colour-gamuts are different and are roughly in the following relationship: 


\section{CIE1931 $\subset$ RGB $\subset$ CMYK $\subset$ SpectralHues (= rainbow)}

Thus in principle colours may exist which are not inside RGB-cube but are present in CIE1931 - space. If an extra RGB - colour was distinguished by a human observer from any monitor-colour, this will enhance reduced RGB space. Attribute reduced would mean a restriction of RGB to pairwise and subjectively discriminated colours. It is paradoxical that monitors display rich CIE-diagrams rather well.

- (2\#)Several colour illusions point to contexts which induce perceptual difference of two, physically identical colour stimuli: Bezold-effect, simultaneous or successive chromatic contrast illusion, Mach-bands, spreading, assimilation or shift of colours etc. The related illusions or conditions may be so intensive that are not neutral in definition of colours, hues or colour constancy.

- (3\#)Objectively different RGB-colours may appear to be identical. This is mainly a threshold problem. However, discrimination threshold may depend on sophisticated conditions. E.g. additive inverses of undistinguished colours can be discriminated.

Colour counting is here approached in RGB-cube. Piecewise experimental comparisons of all pairs of $n=2^{24}$ colours is impossible since involves $2^{47}$ steps. So tricks for easier mass-comparison. should be elaborated. Colour ordering principle are needed and are possible through various mathematical considerations.

\section{Methods}

Image manipulation, coloured spectra and numerical data beyond were generated by own program-codes written in WolframMathematica-5.

\section{Sets of 1D or 2D transitional spectra. 3D colour domains of $R G B$ cube}

In any colour-system ordered colour sets are defined. Most often, the solar spectrum is used by closed into colour circles or wheels. In true solar spectrum the control principle is the wavelength. Transitions between colours are mostly determined by the rainbow. In colour charts, homogenous groups of colours are tabulated, often by ad hoc principles. Inside groups brightness, or saturation usually change. Complete account of all colours is not guaranted.

It seems an economic way to walk through the RGB - cube or on its surface in the hope to compress the whole into a smaller body of the subjectively distinct RGBcolours.

Interesting 1D colour-transitions arise between 2 saturated primary colours, or between a colour and its additive complement, or between a primary and a secondary colour or between two arbitrary colours A and B which are not necessarily neighbors in solar spectrum or in cube. Examples are given in Figure 1. All of these 1D or 2D transitional spectra can be in principle compressed by eliminating undistinguished cases of RGB colours. 


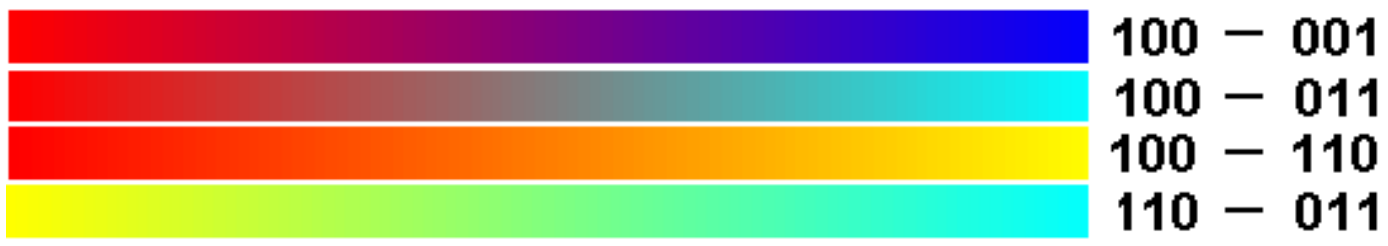

Figure 1. Spectral 1D-transitions between 2 RGB colours. Examples

Various 1D, 2D or 3D wandering strategies over the RGB-cube can be chosen. The colour - points are 3D vectors in a unit-cube or discrete lattice points in a cube with side-length $=255$ integer, withthe big grey diagonal between $\{0,0,0$,$\} and \{255,255$, 255 points. Minings or scans inside cube can be based either on geometrical or even combinatorial principles..

A sensitive point is that close points in Euclidean sense may be close or distant as subjective colours. To get an impression see the 6 sides of this colour cube in Fig. 2.

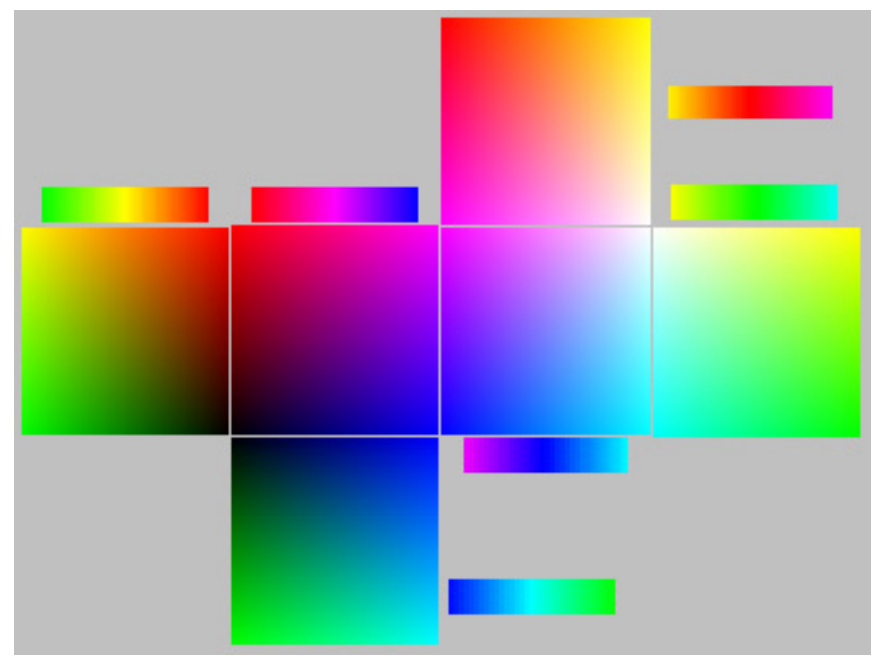

Figure 2. The 6 sides of the RGB colour cube. Skin of RGB-cube with homogenous zones. Each plane corresponds to a $2 D$-spectrum, reducible to $1 D$ spectra. Surface contains more than 390000 colours. Only a few hundred subjectively distinguished.

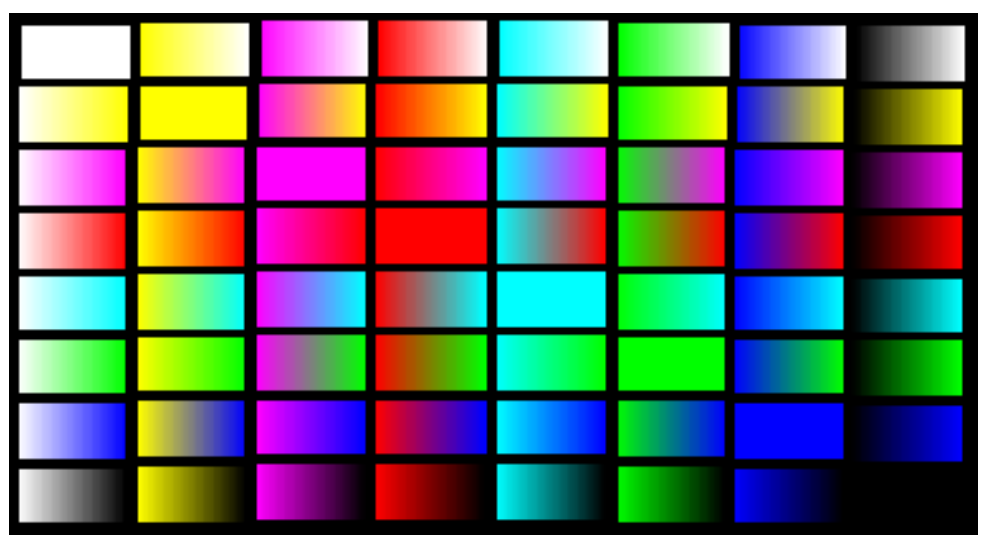

Figure 3. Matrix of all possible $1 D$ transitions spectra between primary and secondary $R G B$ colours. 64 edges and diagonals between 8 corners of $R G B$ cube. Largest cube-diagonal is the grey-scale. Diagonal of matrix includes the 8 constant corner-colours . 
A great number of possible 3D-stratagies of wandering are useful. E.g. cut seemingly colour - uniform subsets like $\{(\mathrm{r} \pm \varepsilon, \mathrm{g} \pm \varepsilon, \mathrm{b} \pm \varepsilon)\}$ even with several thousands of formally different RGB-points, but with only one or a few subjectively monochromous set of pixels.

\section{RGB colours as partitions}

This sorting of RGB-colours is radically different from solar spectral transitions as mostly applied previously. RGB-colours are 3D vectors of integer coordinates between 0 and 255. Their linear (=1D) arrangement is possible only in lexicographic manner violating continuum inside the cube. Listing sorted partitions means a reshuffling of geometrical neighbourhoods.

\section{Formal combinatorial background or model}

Distribute $\mathrm{n}$ cards between three persons, each one receives $0,1, \ldots, \mathrm{n}$ cards. If persons are not distinguished then the solution is the set of partitions of $n$ into 1,2 or three parts. For $\mathrm{n}=10$ we get the following 14 distributions:

$\{\{10,0,0\},\{9,1,0\},\{8,2,0\},\{8,1,1\},\{7,3,0\},\{7,2,1\},\{6,4,0\},\{6,3,1\},\{6,2,2\}$, $\{5,5,0\},\{5,4,1\},\{5,3,2\},\{4,4,2\},\{4,3,3\}\}$

The number of solutions $\mathrm{p}(\mathrm{n})$ is well known and it is a non-trivial integer [11]: $p(n)=\{1,2,3,4,5,7,8,10,12,14,16,19,21,24,27,30,33,37,40,44,48,52,56,61,65$, $70,75,80,85,91,96,102\}$.

As soon as persons are distinguished, more cases arise. If $n=10$, the 66 ordered partitions

(called also as compositions) are as follows:

$\{0,0,10\},\{0,1,9\},\{0,2,8\},\{0,3,7\},\{0,4,6\},\{0,5,5\},\{0,6,4\},\{0,7,3\},\{0,8,2\},\{0,9,1\}$, $\{0,10,0\},\{1,0,9\},\{1,1,8\},\{1,2,7\},\{1,3,6\},\{1,4,5\},\{1,5,4\},\{1,6,3\},\{1,7,2\},\{1,8,1\}$, $\{1,9,0\},\{2,0,8\},\{2,1,7\},\{2,2,6\},\{2,3,5\},\{2,4,4\},\{2,5,3\},\{2,6,2\},\{2,7,1\},\{2,8,0\}$, $\{3,0,7\},\{3,1,6\},\{3,2,5\},\{3,3,4\},\{3,4,3\},\{3,5,2\},\{3,6,1\},\{3,7,0\},\{4,0,6\},\{4,1,5\}$, $\{4,2,4\},\{4,3,3\},\{4,4,2\},\{4,5,1\},\{4,6,0\},\{5,0,5\},\{5,1,4\},\{5,2,3\},\{5,3,2\},\{5,4,1\}$, $\{5,5,0\},\{6,0,4\},\{6,1,3\},\{6,2,2\},\{6,3,1\},\{6,4,0\},\{7,0,3\},\{7,1,2\},\{7,2,1\},\{7,3,0\}$, $\{8,0,2\},\{8,1,1\},\{8,2,0\},\{9,0,1\},\{9,1,0\},\{10,0,0\}$

The number of such partitions corresponds to the well-known triangular numbers [12]:

$P(n)=\{1,3,6,10,15,21,28,36,45,55,66,78,91,105,120,136,153,171,190,210$, $231,253,276,300,325,351,378,406,435,465,496,528,561, \ldots ., 32896, \ldots, 286146\}$, from $\mathrm{n}=0,1, \ldots, 255, \ldots, 755$

If $\mathrm{n}$ cards are divided into 3 persons then the number of such partitions is:

$$
\mathrm{P}(\mathrm{n})=(\mathrm{n}+2)(\mathrm{n}+1) / 2=\mathrm{C}(\mathrm{n}+1,2)
$$

\section{Applications triangular partitions to describe $R G B$ colour vectors}

Partitions as RGB colours are possible for $\mathrm{n}<255+255+255=765$. At $\mathrm{n}>255$, formula of $\mathrm{P}(\mathrm{n})$ given in (Eq. 1.) requires corrections. Colours satisfying $\mathrm{r}+\mathrm{g}+\mathrm{b}=\mathrm{n}$ are in planes of RGB-cube with identical brightness and all colours in this plane are different because $\mathrm{r} / \mathrm{g}$ and $\mathrm{g} / \mathrm{b}$ color ratios are different. Corrected number of colours is $\mathrm{R}(\mathrm{n})=\mathrm{P}(\mathrm{n})-\mathrm{w}(\mathrm{n})$, where $\mathrm{w}(\mathrm{n})$, the number of impossible RGB-partitions which increases rapidly with $\mathrm{n}$ : 
$\mathrm{w}(\mathrm{n})=0$ for $\mathrm{n}<256, \mathrm{R}(\mathrm{n})=0$ if $\mathrm{n}>765$. Number of colours is maximal in the $\mathrm{r}+\mathrm{g}+\mathrm{b}=$ 383 plane and it is 49152 . E.g. $(170,150,190)$ is a legitimate, while $(300,10,200)$ is an invalid RGB-partition of sum-total $=510$ because individual coordinates in cube do not exceed 255. Sequence [13] is involved.

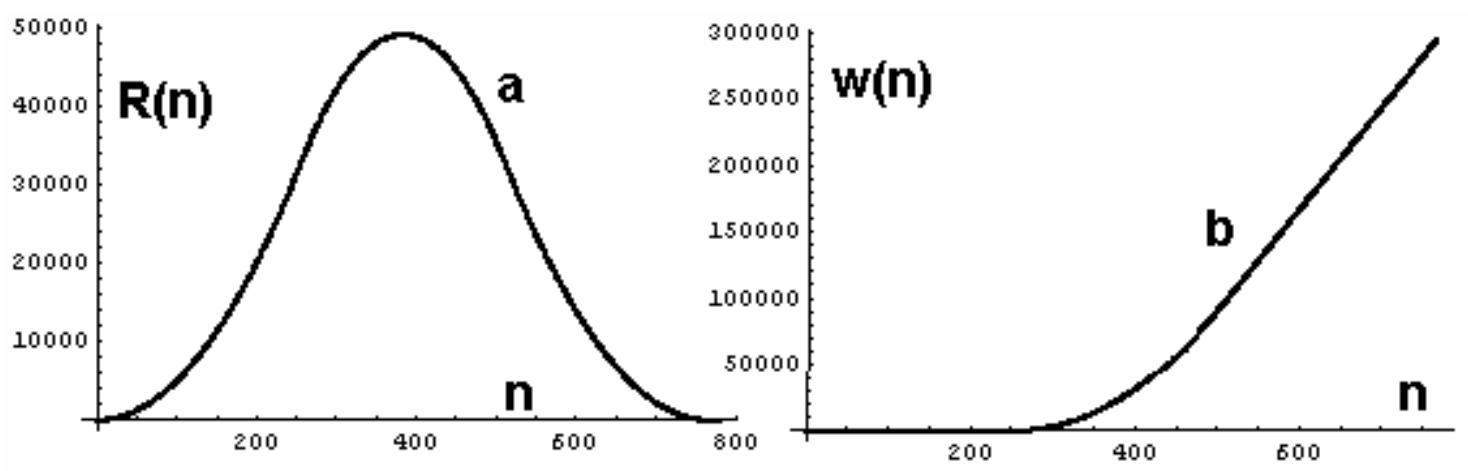

Figure 4. (a) is the number of valid RGB partitions or different colours, (b) the number of invalid partitions in the 766 luminosity layers of $R G B-$ cube orthogonal to and around the big grey diagonal axis. Plotted against $n=r+g+b$.

In a valid RGB partition all components are $\leq 255$. Partition of $\mathrm{n}$ means luminosity $=$ $\mathrm{n}$. Among the R(n) RGB-solutions for $\mathrm{r}+\mathrm{g}+\mathrm{b}=\mathrm{n}$, pair of ratios $\left\{\mathrm{k}_{1}=\mathrm{r} / \mathrm{g}, \mathrm{k}_{2}=\mathrm{r} / \mathrm{b}\right\}$ are all different and characterizes colours. Physically these ratios describe emission ratios for a given coloured pixel. Per analogiam, in retina the three cones were activated - or absorb energy or quanta according to analogous characteristic ratios. These two interpretations obviously require further corrections and harmonisation by application of precise emission and absorption spectra of monitor or cones respectively.

- Example 1: $\mathrm{n}=255$; initial colour $=(85,111,59)$, is a relatively dark olive-green The brightest integer version is $(170,222,118)$.

- Example 2: $\mathrm{n}=6$; initial colour $=(3,2,1)$ is an almost black with invisibly weak reddish/violet shade. Max $[\{3,2,1\}]=3$, thus $255 / 3=85$ colour-shades with invariant $\mathrm{r} / \mathrm{g}, \mathrm{g} / \mathrm{b}$ ratios are generated. Spectra are displayed in (Fig. 5) Terminal colour is $(255,85,170)$. In the examples ratios are kept invariant, lightness are increases.

Figure 5. See in text above. Example 1 and 2

\section{Partitional spectra}

Given $\mathrm{r}+\mathrm{g}+\mathrm{b}=\mathrm{n}$. in RGB-space. It is a plane with constant brightness and orthogonal to the big grey diagonal of RGB-cube. When ( $\mathrm{r}, \mathrm{g}, \mathrm{b})$ vectors run through all partitions of $\mathrm{n}$ and the partitions are lexicographically sorted, then we get a partition spectrum (p-spectrum) with resolution $1 / \mathrm{n}$ or with lightness $\mathrm{n}$. If $\mathrm{n}$ is small, then all generated colours are shaded, darkish. If $\mathrm{n} \leq 255$, the length of a $\mathrm{p}$-spectrum, i.e. its number of colours is $(n+2)(n+1) / 2$. At $n=\{0,1,2,4,8,16,32,64,128,255\}$ these lengths 
are $\{1,3,6,15,45,153,561,2145,8385,33153\}$ respectively. Inside these spectra at least one of the ratios of $\{\mathrm{r} / \mathrm{g}, \mathrm{r} / \mathrm{b}\}$ and consequently $\mathrm{g} / \mathrm{b}$ change with particular partitions from $(0,0, n), \ldots$ to $(n, 0,0)$ i.e. with individual colours as we define here. The spectrum starts with RGB primary blue and terminates with primary red. In order to get brighter spectral colours, all channel coefficients were further divided by the maximum of $\{\mathrm{r}, \mathrm{g}, \mathrm{b}\}$ components. Consequently, constancy of brightness disappears, while colour-character and ratios remain invariant. In each colour vector at least one coefficient will have the magnitude 1 (or 255). Therefore colours are projected onto the lighter half-surface of RGB-cube.

These partition spectra show remarkable properties. 1\#. - With increasing $\mathrm{n}$, partition - spectrum includes $\mathrm{n}$ colour bands. 2\#. - Inside a band and also along consecutive bands, regular transitions are observable, bands shorten. 3\#. - First half or so bluish greenish alternation is visible. In the second half, orange-red becomes overwhelming. 4\#. At $n=1$ and at $n=3$ only the well-known RGB primary and nearly secondary colours (red, green, blue, yellow, magenta, turquoise) emerge. 5\#. Colours proximal to white appear in the first series of $(1, \mathrm{x}, \mathrm{y})$ colours, while black shades arise among inverted $(0, \mathrm{x}, \mathrm{y})$ ones (Fig. 6b, Fig. 6b1).

Partition spectra list all distinct colours with 1/n upper bound for deviation of colourcoefficient-ratios. It sorts but did not bring close enough subjectively close colours. Further transformation should be introduced in order to classify colours (not hues!). This better rearrangement was carried out by their computed hues as an HSV-parameter. Results see in (a1), (b1) of (Fig. 6.)

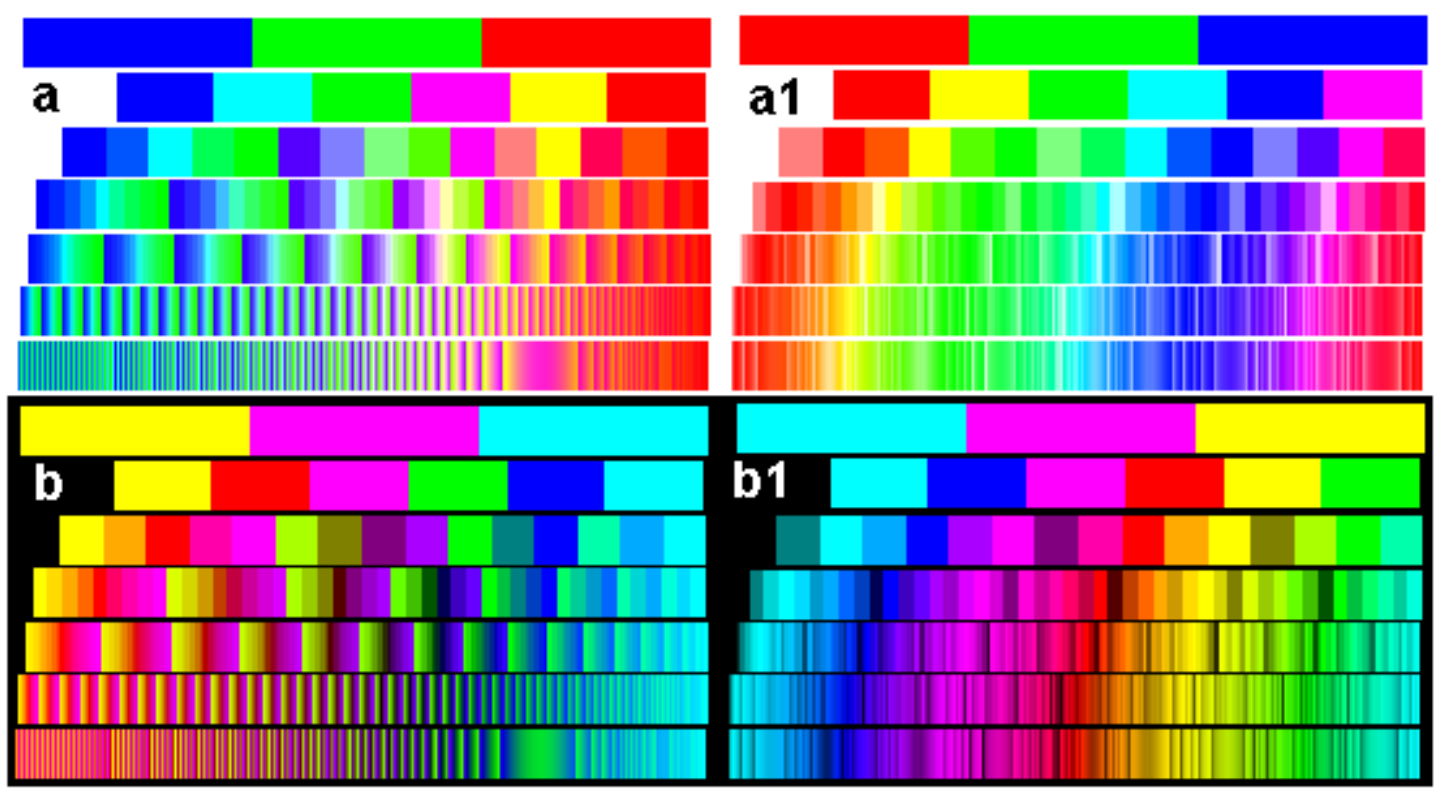

Figure 6. (a) $=$ Partition spectra with increasing resolutions $n=1,2, \ldots, 64 ; \max [r, g, b]=1$. (b) $=$ Spectra of (a) are inverted, $\min [r, g, b]=0$. In each spectrum number of colour is $(m+2)(m+1) / 2$. Spectrum (al) and (b1) rearranged of (a) and (b) according to magnitude of their computed HSV hues-angles 


\section{A few details of various partition spectra}

Partition spectra at different values of $\mathrm{n}$ (Fig. 6 ) are similar to each other. Each starts with BTG (blue, turquoise, green) bands. It lasts to $30-40 \%$ of the total length. Medial $20 \%$ zone keeps green with viola and white. In $3^{\text {rd }}$ zone the bands are composed of lilacmagenta-yellow while green disappears. Finally the bands are dominated with red orange - yellow. (See Fig. 7.) To reach significant colour-number-reduction, comparative psychophysical test of the bands seem here fruitful but are still difficult. Only the completeness of partition-colours is sure.

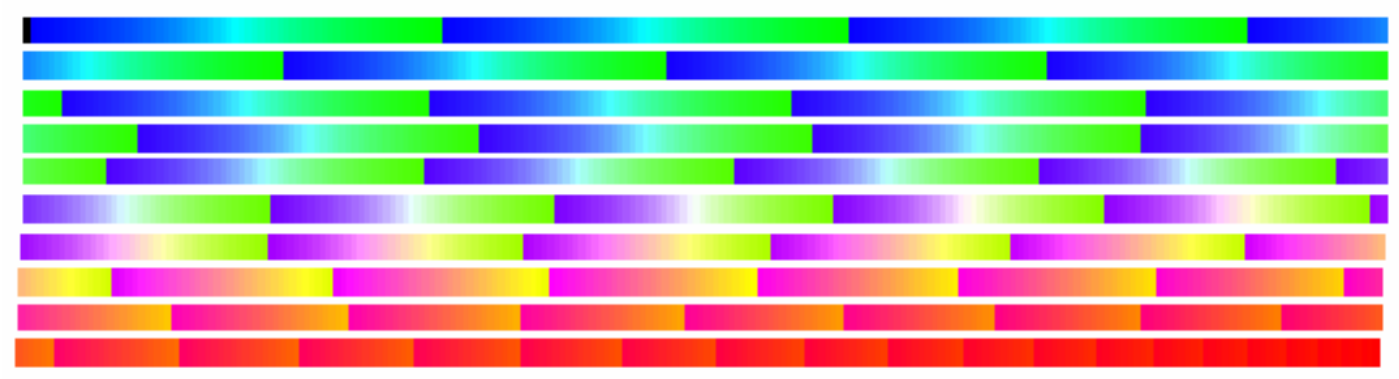

Figure 7. Single partition spectrum with resolution $n=64$. It consists 2145 RGB colours. Observe regular metamorphoses of the 64 bands.

\section{Hues along partition spectra. Conversions to HSV colour system}

Several conversion formula from RGBcolours to HSV colour-representation exist. HSV colour - space [5] describes a colour as a (hue, saturation, value) $=(\mathrm{H}, \mathrm{S}, \mathrm{V})$ vector. For a given RGB- point, the parameter $\mathrm{V}$ is either the maximun or arithmetical mean of RGB coordinates. Saturation S, is either ( $\max -\min ) / \max$ or standard deviation of RGB coordinates. To compute component H of HSV the most often used formula is applied here:

$$
\mathrm{H}=\mathrm{b}+60\left(\mathrm{c}_{1}-\mathrm{c}_{2}\right) /(\max -\min )
$$

Here $c_{1}-c_{2}$ is $\mathrm{G}-\mathrm{B}, \mathrm{B}-\mathrm{R}, \mathrm{R}-\mathrm{G}$ or $\mathrm{b}=0,2,4$ if $\max \{\mathrm{R}, \mathrm{G}, \mathrm{B}\}=\mathrm{R}, \mathrm{G}$, or $\mathrm{B}$ respectively. The result is a hue expressed by an angle in degree between 0 and 360 , where red $=0$, green $=120$, blue $=240$. It is hard to believe that this concept of hue corresponds to wavelengths with one $\mathrm{nm}$ accuracy.It is a $2^{\text {nd }}$ definition of hue. 


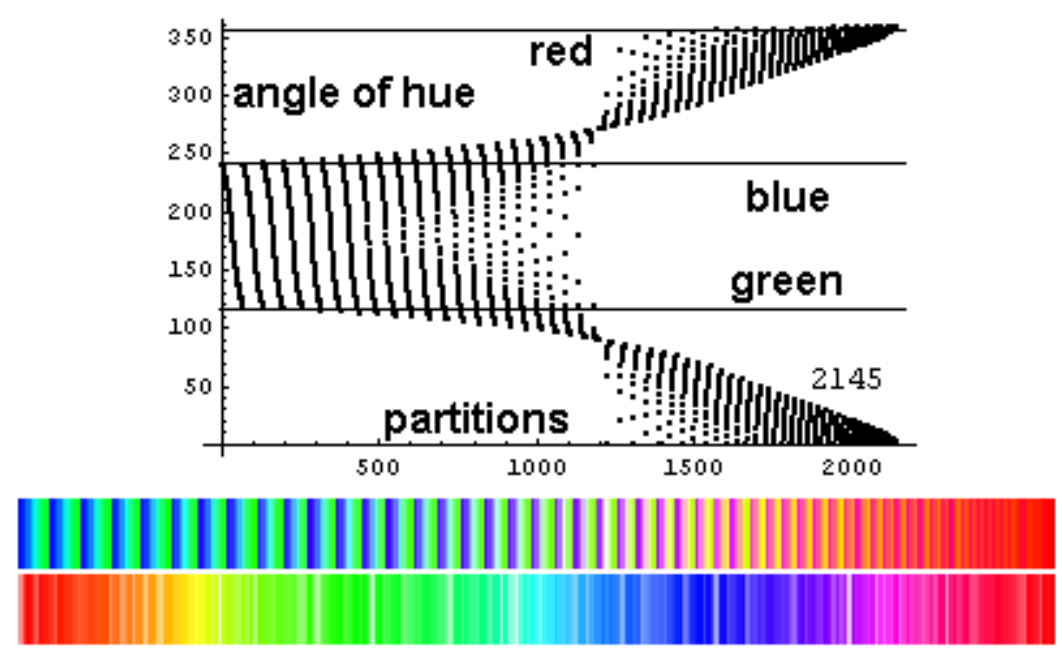

Figure 8. Hues in degrees of HSV - system plotted against 2145 RGB -colours sorted as partitions of 64. Upper: partition spectrum. Lower pseudo solar spectrum is obtained by reshuffling partition - colours by their computed hues.

\section{Conclusions}

The aim was here to get closer to the number of colours distinguished by a normal human observer. Long history [14] and branches of colour science [15, 16] all suggest that despite of advanced technics available, the most incoherent, diverse solutions are still proposed. Answer is more diversified than it was justified. One possible cause of this uncertainity is the lack of suitable arrangement of colours which would permit the complete and repetition-free survey of a color-space. Here, various geometrical and combinatorial ordering strategies based on sophisticated subsets of RGB-cube were proposed.. E.g. linear arrangement by partitions i.e. also by intensity ratios of RGBprimary channels may achive a regularity, brings closer subjectively similar colours. Only construction of further order algorithms makes worthy to start psychophysical testing identity and difference of colour species.

RGB partition-lists give colour spectra with regularly but still capriciously dispersed colours, albeit these lists are complete with respect of ratios or other aspect. When formal HSV-hues are computed for a given partition sequence or set of other RGBcolours, then this sequences can be simultanously reshuffled by HSV-hues computed with help of (Eq. 2). What we obtain, it is a complete or partial pseudo-solar spectrum of colours (not that of hues!). In Fig. 8, the lower spectrum was computed and rearranged from the upper partition spectrum. Also in Fig. $6 a 1$ and $6 b 1$ the satellite pseudo-solar spectra are obtained from various partition spectra.

Such a rearrangement can be done with an arbitrary initial colour-list as follows: (1\#) - determine (r, g, b) for each colour. (2\#) - Compute $h=\eta[r, g, b]$ sizes of hueangles. (3\#) - Arrange $h$ values by their magnitude. List the original and corresponding RGB-colour arguments vectors by this new order of their hues. (4\#) - Compute and display the new colour-spectrum.

Close colours get physically closer. Now you can start counting colours or you can discriminate with real hope and with efficiency. In pseudo-solar-spectra the similare colours are physically closer along the new 1D arrangement as they were before. 
We emphasize that any colour census is at last a psychophysiological task but impossible to carry out without such preparatory transformations. This work has been initiated.

The more conservative way to reduce colour cube is to generate seemingly uniform RGB colour fields. Count number of its distinct colours. By this way local reduction factors can be collected. E.g. very often in domains with 1000-5000 distinct RGBcolours 12 or 3 colours can be subjectively distinguished.

Obviously, several issues are out of our scope. We did not deal with standardization problems related to RGB-cube or monitors, neither with color vision or color blindness. The aim and results are in direct relationships only with and are restricted to those conceptual frameworks which make easier at all any efficient colour census. Better and computerizable colour mining methods inside a colour space chosen for analysis may diminishes gap between 25000 and 250000 our actual rough guesses.

\section{Glossary}

CIE1931 = Comission International d'Eclarage from 1931.

$\mathrm{RGB}=$ Red - Green - Blue colour system for monitor

HSV = Hue, Saturation, Value or Lightness

CMYK = Cyan - Magenta -Yellow - Black for printers

Acknowledgements. Grateful thanks to M.Csati for valuable help. Grants were supplied by MTA-TKI.

\section{REFERENCES}

[1] Wasserman, G.S. (1974): Colour Vision: An Historical Introduction. - WileyInterscience, New York.

[2] Wright, W.D. (1967): The Rays are Not Coloured. - Adam Hilger Ltd, Bristol.

[3] Lukács Gy. (1982): Színmérés. - Müszaki Könyvkiadó, Budapest

[4]---Nemcsics A. (2004): Színdinamika. - Akadémiai Kiadó, Budapest.

[5]---Smith, A.R. (1978): Colour gamut transform pairs. - Computer Graphics 12(3): 12-19.

[6]---Wright, W.D. and Pitt, F.H.G. (1934): Hue-discrimination in normal colour-vision. Proc. Phys. Soc. 45: 459-473.

[7]---Wright, W.D (1946): Researches on Normal and Defective Colour Vision. - Henrz Kimpton, London.

[8]---Pitt, F.H.G. (1944): The Nature of Normal Trichromatic and Dichromatic Vision. Proc.R.Soc. Ser B. 132: 101-117.

[9]---Krudy, A., Ladunga K. (2001): Measuring Wavelength Discrimination Threshold Along the Entire Visible Spectrum. - Periodica Polytechnica Ser. Mech. Eng. 45: 41-48.

[10] -MacAdam D.L. (1942): Visual sensitivities to colour differences in daylight. - J. Opt. Soc. Am. 32: 247.

[11] -http://www.research.att.com/projects/OEIS?Anum=A000217, triangular numbers.

[12] -http://www.research.att.com/projects/OEIS?Anum=A001399, number of partitions into at most 3 parts.

[13] -http://www.research.att.com/projects/OEIS?Anum=A045943, special triangular numbers.

[14] -Sherman P.D. (1981): Colour Vision in the Nineteenth Century. The Young-HelmholtzMaxwell Theory. - Adam Hilger Ltd, Bristol. 
[15] -Wyszecki, G., Stiles, W.S. (1967): Colour Science, Concepts and Methods. - WileyInterscience, New York.

[16] -Judd, D. B., Wyszecki, G. (1963) : Color in Business, Science, and Industry. - WileyInterscience, New York. 